\title{
Perfil dos portadores de hepatite A no Estado do Rio de Janeiro, no período de 2010
}

\section{a 2018}

\author{
Profile of hepatitis carriers in the State of Rio de Janeiro, from 2010 to 2018 \\ Perfil de los titulares de hepatitis en el Estado de Rio de Janeiro, de 2010 a 2018
}

Recebido: 23/03/2021 | Revisado: 29/03/2021 | Aceito: 01/04/2021 | Publicado: 04/04/2021

\author{
Natália Coelho Cavalleiro dos Santos \\ ORCID: https://orcid.org/0000-0001-6209-4645 \\ Universidade Federal do Estado do Rio de Janeiro, Brasil \\ E-mail: nataliacavalleiro@yahoo.com.br \\ Luciane de Souza Velasque \\ ORCID: https://orcid.org/0000-0002-4269-4755 \\ Universidade Federal do Estado do Rio de Janeiro, Brasil \\ E-mail: luciane.velasque@uniriotec.br \\ Bianca Ramos Marins Silva \\ ORCID: https://orcid.org/0000-0002-2664-9794 \\ Universidade Federal do Estado do Rio de Janeiro, Brasil \\ E-mail: bianca.silva@unirio.br
}

\begin{abstract}
Resumo
Objetivo: Este estudo busca caracterizar o perfil dos indivíduos com hepatite A, no Estado do Rio de Janeiro, no período de 2010 a 2018. Metodologia: Trata-se de uma pesquisa transversal desenvolvida na abordagem quantitativa. Foram utilizados dados secundários provenientes da Ficha de Notificação das Hepatites Virais do banco do Sistema de Informação de Agravos de Notificação, cedida pela Secretaria Estadual de Saúde do Rio de Janeiro. Resultados: Foram analisados 48672 notificações, sendo que 3734 foram notificações confirmadas de portadores exclusivo da hepatite A, 28719 referentes a outras hepatites, 614 de co-infecção por mais de uma hepatite viral e 15605 ignoradas. A maior prevalência da hepatite A foi encontrada nos indivíduos com 4 a 8 anos de estudo, da raça negra, faixa $\leq 24$ anos e sendo água e alimentos contaminados a principal fonte de contágio. Conclusão: A notificação dos casos e o preenchimento adequado é importante para definições de ações sanitárias capazes de mitigar os danos à saúde pública.

Palavras-chave: Hepatite A; Notificação; Prevalência.
\end{abstract}

\begin{abstract}
Objective: To characterize the profile of individuals with hepatitis A in the State of Rio de Janeiro, from 2010 to 2018. Methodology: This is a cross-sectional research developed in the quantitative approach. Secondary data from the Viral Hepatitis Notification Form of the Bank of the Notifiable Diseases Information System, provided by the State Health Secretariat of Rio de Janeiro, were used. Results: 48672 notifications were analyzed, of which 3734 were confirmed notifications of hepatitis A carriers only, 28719 were related to other hepatitis, 614 were co-infected with more than one viral hepatitis and 15605 were ignored. The highest prevalence of hepatitis A was found in individuals with 4 to 8 years of study, black, aged $\leq 24$ years and with contaminated water and food being the main source of contagion. Conclusion: Notification of cases and proper completion is important for the definition of health actions capable of mitigating damage to public health.
\end{abstract}

Keywords: Hepatitis A; Notification; Prevalence.

\section{Resumen}

Objetivo: Este estudio busca caracterizar el perfil de individuos con hepatitis A, en el estado de Río de Janeiro, de 2010 a 2018. Metodología: Se trata de una investigación transversal desarrollada en el enfoque cuantitativo. Se utilizaron datos secundarios del Formulario de Notificación de Hepatitis Virales del Banco del Sistema de Información de Enfermedades Notificables, proporcionado por la Secretaría de Salud del Estado de Río de Janeiro. Resultados: Se analizaron 48672 notificaciones, de las cuales 3734 fueron notificaciones confirmadas de solo portadores de hepatitis A, 28719 estaban relacionadas con otras hepatitis, 614 estaban coinfectadas con más de una hepatitis viral y 15605 fueron ignoradas. La mayor prevalencia de hepatitis A se encontró en individuos de 4 a 8 años de estudio, negros, $\leq 24$ años y con agua y alimentos contaminados como la principal fuente de contagio. Conclusión: La notificación de casos y su debida cumplimentación es importante para la definición de acciones de salud capaces de mitigar los daños a la salud pública.

Palabra clave: Hepatitis A; Notificación; Prevalencia. 


\section{Introdução}

A hepatite A (HAV) é uma infecção causada por um vírus hepatotrófico, no qual o homem é o principal reservatório com relevância epidemiológica. O vírus pertence à família Picornaviridae, sendo sua via de transmissão feco-oral. O contágio está associado a precariedade do saneamento básico, higiene pessoal, qualidade da água e dos alimentos. (Ministério da Saúde, 2017)

Segundo o Boletim Epidemiológico, no período entre os anos 1999 a 2019, foram notificados pelo Sistema de Informação de Agravos de Notificação (SINAN), 673.389 casos confirmados de hepatites virais no Brasil. Entre esses casos, $168.036(25,0 \%)$ são referentes aos casos de hepatite A, $247.890(36,8 \%)$ aos de hepatite B, $253.307(37,6 \%)$ aos de hepatite C e $4.156(0,6 \%)$ aos de hepatite D. (Brasil, 2020)

Os casos confirmados de hepatites virais, por meio da "Ficha de Investigação das Hepatites Virais", devem ser notificados e registrados no SINAN, em até sete dias. Sendo as principais fontes notificadoras: unidades de saúde, hemocentros e bancos de sangue, clínicas de hemodiálise, laboratórios, comunidade, escolas e creches, dentre outras. (Ministério da Saúde, 2017)

Assim, a notificação em saúde possui o intuito de orientar, acompanhar e avaliar os modelos de atenção à saúde e das ações de prevenção e controle de doenças, sendo a base para a gestão em saúde (Araújo \& Silva, 2015). Para Bandeira (2017), entre os casos agudos por hepatite A é possível observar que indivíduos infectados por HAV são 4 vezes maior quando comparado aos casos agudos de hepatite $\mathrm{B}$ e mais de 10 vezes superior às notificações de casos agudos de hepatite $\mathrm{C}$. Conforme Chaves, Castro e Oliveira (2017), no Brasil, as hepatites virais, tornaram-se uma doença de notificação compulsória, em 1996, assim, possibilitando conhecer a magnitude e as características epidemiológicas da doença no país.

Segundo Guimarães et al. (2019), o vírus da hepatite A atinge a humanidade desde os primeiros seres vivos, e a partir do surgimento de comunidades, e o convívio de pessoas em grupos, o que favoreceu a transmissão sustentada do HAV, no entanto, a doença foi caracterizada no ano de 1973, pelos pesquisadores americanos Stephen Feinstone, Albert Kapikian e Robert Purcell. A forma de contágio da doença ocorre por via oral, através da ingestão do HAV com alimentos ou água contaminados. O vírus da hepatite A possui distribuição universal, sendo a sua prevalência associada principalmente em regiões onde o grau de higiene e os recursos sanitários disponíveis não são satisfatórios para a população (Pereira \& Gonçalves, 2003). Ademais, o HAV pode sobreviver períodos de 12 semanas ou até 10 meses, na água ou ser retido por moluscos ou crustáceos e acumular o vírus até 15 vezes mais do que o nível original da água. (Ferreira \& Silveira, 2004)

Em estudo realizado no norte de Minas Gerais, no período de 2001 a 2006, foi identificado aumento importante nas notificações das hepatites virais, porém a hepatite A foi a mais incidente (64,7\% dos casos), seguida pelas hepatites B (17,8\%) e C $(2,4 \%)$. O estudo também revelou que crianças em idade escolar são mais suscetíveis a contraírem hepatite A. (Ferreira, Gonçalves \& Gonzaga, 2017)

Segundo dados do Instituto Brasileiro de Geografia e Estatística (IBGE), em 2008 o estado do Rio de Janeiro possuía a terceira melhor posição, entre as unidades federativas, com 49,2\% dos seus domicílios com acesso a esgotamento sanitário. Porém, o esgoto despejado no ambiente sem tratamento ainda é significativo, sendo responsável por levar contaminação e disseminar a hepatite A. (Bandeira, 2017)

Nas Américas e na Europa foram reportados episódios de surto de hepatite A por via sexual, apesar de não ser uma via comum de transmissão. Contudo, pessoas que foram infectadas pelo HAV apresentam imunidade para o vírus, mas permanecem suscetíveis as outras hepatites. (Ministério da Saúde, 2018)

Desta forma, a prevalência do vírus da hepatite A serve como indicador da precariedade do saneamento básico e a qualidade da água. Entre as 27 capitais brasileiras, foi observado que 11 apresentaram taxas de incidência do HAV superior à média nacional (0,4 casos por 100 mil habitantes), em ordem decrescente, por taxa de incidência do vírus, o Rio de Janeiro 
ocupa o sexto lugar, com 0,9. (Brasil, 2020). O HAV é de caráter benigno na maioria dos casos, sendo que em menos de $1 \%$ dos casos, geralmente em indivíduos com mais de 65 anos de idade, evolui para a insuficiência hepática aguda grave, apesar da sua forma crônica não ocorrer. (Brasil, 2009)

Diante disto, o presente estudo tem como objetivo analisar os dados disponíveis no Sistema de Informação de Agravos de Notificação do Ministério da Saúde (SINAN/MS) relacionados as notificações do estado do Rio de Janeiro, no período de 2010 a 2018, buscando identificar o perfil sócio econômico da população acometida pela hepatite A, as principais fontes de contaminação e identificar ainda a existência de associação entre os fatores socio econômicos dos indivíduos expostos ao vírus da hepatite A com as outras hepatites virais; os co-infectados e quantificar as notificações ignorada. Desta forma, será possível compreender o perfil da população acometida pela infecção causada pelo vírus da hepatite A e estimular futuras pesquisas que proporão estratégias sanitárias capazes de mitigar os danos à saúde coletiva.

\section{Metodologia}

Trata-se de um estudo do tipo transversal, de abordagem quantitativa, onde o fator de exposição e o desfecho são observados no mesmo momento histórico (Bordalo, 2006). Segundo Martins Júnior (2017), a análise quantitativa dos dados é a quantificação dos resultados que foram coletados sob a forma de símbolos matemáticos e/ou estatísticos.

Os dados secundários sobre hepatite A são oriundos da Ficha de Notificação das Hepatites Virais, os quais foram provenientes do banco de dados do SINAN, cedida pela Secretaria Estadual de Saúde do Rio de Janeiro, no período de 2010 a 2018.

Para este estudo, as variáveis sociodemográficas, selecionadas foram: idade (agrupada em; $\leq 24$ anos, 25-39 anos, 40-59 anos e $\geq 60$ anos), sexo (masculino e feminino), escolaridade (agrupada conforme os anos completos de estudo: até 4 anos de estudo, 4 a 8 anos de estudo, 8 a 12 anos de estudo, > 12 anos de estudo e ignorado) e raça (agrupada em raça negra, pardos mais pretos, raça branca e outros, amarelos mais indígenas). O fator "outras raças" (raça amarela e indígena) foi agrupado devido ao número reduzido de casos. Segundo, IBGE a raça negra é definida por pessoas pardas e pretas. (Barrocas, Moraes \& Sousa, 2019)

Ademais, foi analisada a variável provável fonte de contágio (sexual, transfusional, uso de Drogas, vertical, acidente de trabalho, hemodiálise, domiciliar, tratamento cirúrgico, tratamento dentário, pessoa/pessoa, alimento/água contaminado, outros e ignorado).

O processamento dos dados foi realizado por meio do programa de domínio público R (R Foundation for Statistical Computing, versão R-3.5.1) e do Microsoft Excel®.

Para a análise das variáveis qualitativas: escolaridade, provável fonte de contágio, raça, sexo e idade, foi realizado o teste de hipótese Qui-quadrado, considerando o nível de significância menor que 0,5\%.

O referido estudo foi aprovado pelo Comitê de Ética em Pesquisa da Universidade Federal do Estado do Rio de Janeiro - UNIRIO (CEP/UNIRIO), sob parecer de número 3.926.279, e seguiu as normas definidas pela Resolução n. 466/2012 do Conselho Nacional de Saúde.

\section{Resultados e Discussões}

No período de 2010 a 2018 foram analisados 48672 notificações do SINAN, conforme dados cedidos pela Secretaria de Estado de Saúde do Rio de Janeiro, sendo que 3734 (11\%) foram notificações confirmadas de portadores exclusivo da hepatite A, 28719 (84,35\%) notificações referentes a outras hepatites (hepatite B, C entre outras), 614 (1,8\%) notificações de co-infecção por mais de uma hepatite viral e 15605 (32,0\%) como notificações ignoradas. 
Além disso, no mesmo intervalo de tempo citado anteriormente, as notificações para hepatite A no ano de 2010 foram 491 (13,1\%), em 2011 foram 541 (14,5\%), em 2012 foram 707 (18,9\%), em 2013 foram 672 (18\%), em 2014 foram 437 (11,7\%), em 2015 foram 205 (5,5\%), em 2016 foram 25 (0,7\%), em 2017 foram 166 (4,4\%) e em 2018490 (13,1\%) casos. (Gráfico 1)

A cidade do Rio de Janeiro foi responsável por 79\% dos casos notificados (n=4554 notificações) no período de 2007 a 2015, sendo a capital a maior notificadora. Os surtos da doença no Rio de Janeiro, em sua maior parte, ocorreram em instituições fechadas, como creches e escolas, por meio do contato com água contaminada com o vírus ou transmissão pessoapessoa. (Bandeira, 2017)

Gráfico 1 - Distribuição da hepatite A, das co-infecções, outras hepatites e os notificações ignorados, no Estado do Rio de Janeiro, 2010 à 2018.

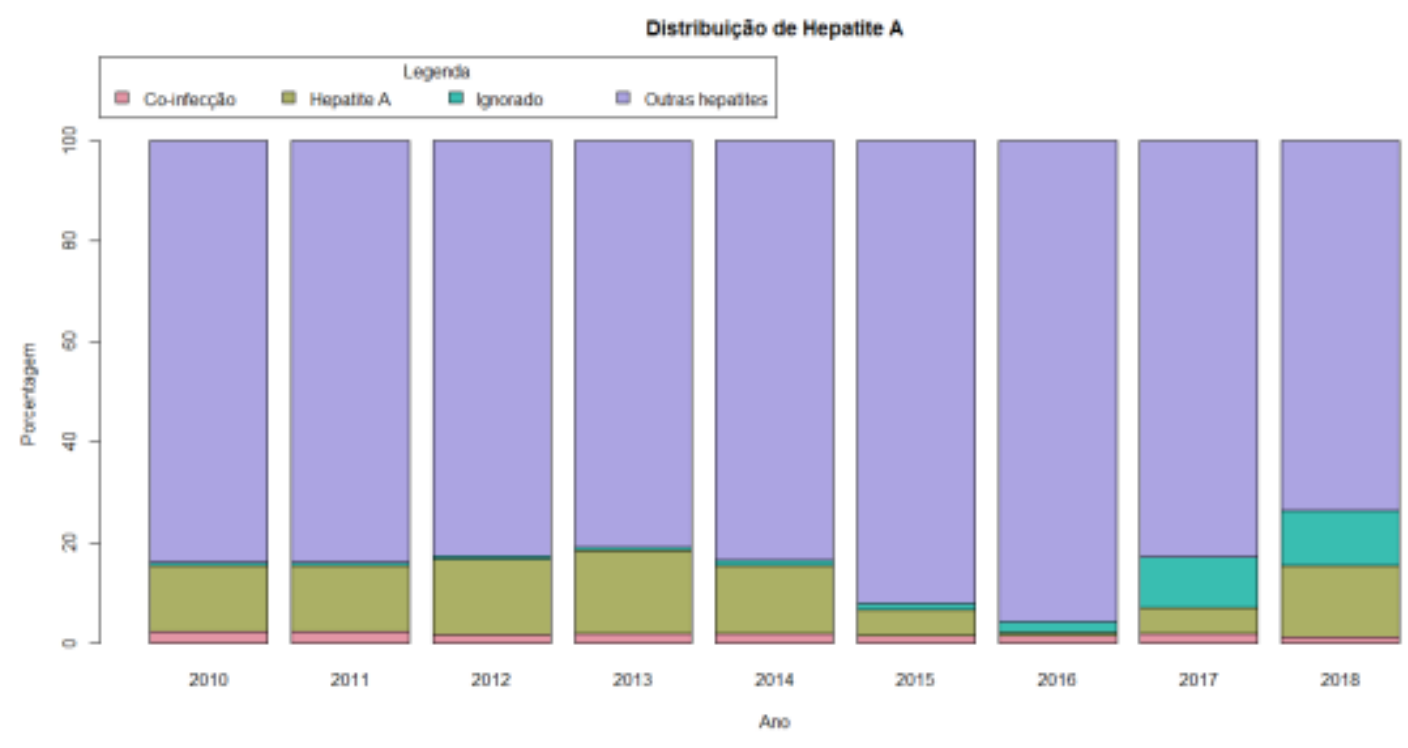

Fonte: Autores.

Vale ressaltar, que a hepatite A está entre as doenças relacionadas ao saneamento inadequado (DRSAI) e que aproximadamente $17 \%$ da população brasileira ainda não possui acesso a água tratada através de rede e metade não possui a coleta de esgoto feita de maneira adequada. (Barrocas, Moraes \& Sousa, 2019)

O município do Rio de Janeiro de Janeiro possui dois prestadores de serviço no tratamento do esgoto, CEDAE/RJ (companhia estadual do subgrupo dos prestadores de abrangência regional) e FABZO/RJ (empresa privada do subgrupo de prestadores de serviços de abrangência local). $\mathrm{O}$ índice médio de atendimento urbano com rede coletora de esgotos aponta valores entre $40 \%$ a $70 \%$. (Brasil, 2019)

Segundo o Sistema Nacional de Informações sobre Saneamento (SNIS) é notório o consumo médio per capita de água no estado do Rio de Janeiro, com 254,9 1/hab./dia em 2018, apresentado valor 39,6\% acima da média da macrorregião Sudeste. Isso pode ser justificado devido aos baixos índices de medição verificados no estado, pois expressiva parcela dos volumes consumidos são estimados. (Brasil, 2019)

O presente estudo identificou que os indivíduos com a faixa etária $\leq 24$ anos apresentaram a maior prevalência de casos reagente ao HAV, com 1776 (57,62\%) notificações, e os indivíduos maiores de 60 anos foram os de menor prevalência, com $135(2,82 \%)$ notificações. (Tabela 1).

Conforme o Boletim Epidemiológico, houve o aumento do número de casos de hepatite A, principalmente na região Sudeste brasileira, entre homens de 20 a 39 anos. Acredita-se que esse aumento está relacionado a práticas sexuais (Brasil, 
2020). Segundo Nunes (2020), a doença tende a ocorrer, prioritariamente, em crianças menores de 10 anos, sendo que o auge da moléstia ocorre entre os 5 e 6 anos de idade.

Ainda, foi verificado uma estabilização, inferior quando comparado aos anos anteriores ao programa de vacinação nas taxas de incidência do HAV em território nacional. Esse fator pode estar associado à epidemia registrada em adultos de 20 a 39 anos, ocorrida em 2017, com destaque para a Região Sudeste, e ao fato de a queda na cobertura vacinal infantil estar ligada à hesitação da vacina. (Brito \& Souto, 2020)

Foi possível observar que a menor taxa de prevalência da doença ocorreu em indivíduos que possuem de 8 a 12 anos de estudo (2,92\%) e a maior ocorreu entre os que possuem de 4 a 8 anos de estudo (7,97\%). Porém, o quantitativo de notificações ignoradas foi significativo, apresentado 1,39\% dos casos. A menor taxa de casos no grupo que possui de 8 a 12 anos de estudo pode estar relacionada a uma parcela da população mais esclarecida sobre a doença e a sua prevenção. (Tabela $1)$.

Em um outro estudo, realizado no Rio de Janeiro, foi encontrado a relação da baixa escolaridade com os acometimentos da hepatite $\mathrm{A}$, sendo (57,87\%) não completaram o ensino fundamental ou são analfabetos (Bandeira, 2017). Tal estudo mencionado anteriormente, diverge com o presente estudo, o que pode ter relação com o número de notificações ignoradas.

Quando analisado a raça/cor da pele, a maioria dos indivíduos reagente ao HAV são da raça negra com 1403 $(11,53 \%)$ dos casos, e a prevalência das notificações ignoradas foi de 1386 (12,31\%) dos casos. (Tabela 1)

Atualmente, grande parte da população brasileira negra vive em condições sanitária precária. Conforme o Censo 2010, 61\% da população sem cobertura do abastecimento de água, 67\% da população sem acesso à coleta dos resíduos e 58\% da população sem cobertura do esgotamento sanitário era negra. (Jesus, 2020) 
Tabela 1 - Distribuição das notificações de hepatite A, conforme idade, os anos de escolaridade e a raça/cor da pele, no Estado do Rio de Janeiro, 2010 à 2018 ( $p$ valor < 0,001).

\begin{tabular}{|c|c|c|c|c|c|c|c|c|c|c|}
\hline \multirow[t]{2}{*}{ Idade } & \multicolumn{2}{|c|}{ Co-infecçào } & \multicolumn{2}{|c|}{ Hepatite A } & \multicolumn{2}{|c|}{ Ignorado } & \multicolumn{2}{|c|}{ Outros* } & \multirow{2}{*}{ Total } & \multirow[t]{2}{*}{ P valor } \\
\hline & $N$ & $\%$ & $\mathbf{N}$ & $\%$ & $\mathbf{N}$ & $\%$ & $\mathbf{N}$ & $\%$ & & \\
\hline$\leq 24$ anos & 9 & $0,29 \%$ & 1776 & $57,62 \%$ & 1282 & $41,60 \%$ & 15 & $0,49 \%$ & 3082 & \\
\hline $25-39$ anos & 98 & $1,24 \%$ & 1224 & $26,10 \%$ & 3391 & $72,30 \%$ & 17 & $0,36 \%$ & 4690 & $<0,001$ \\
\hline 40.59 anos & 185 & $2,78 \%$ & 440 & $6,61 \%$ & 5983 & $89,85 \%$ & 51 & $0,77 \%$ & 6639 & \\
\hline 260 anos & 341 & $7,11 \%$ & 135 & $2,82 \%$ & 4267 & $\$ 9,03 \%$ & 50 & $1,04 \%$ & 4793 & \\
\hline Ignorados & 21 & $2,49 \%$ & 159 & $18,84 \%$ & 663 & $78,55 \%$ & 1 & $0,12 \%$ & $\$ 44$ & \\
\hline
\end{tabular}

\begin{tabular}{|c|c|c|c|c|c|c|c|c|c|c|}
\hline & $N$ & $\%$ & $\mathbf{N}$ & 9 & $\mathbf{N}$ & 96 & $\mathbf{N}$ & 96 & & \\
\hline Ate 4 anos de estudo & 62 & $1,31 \%$ & 423 & $8,92 \%$ & 1201 & $25,32 \%$ & 3057 & $64,45 \%$ & 4743 & \\
\hline 4 a 8 anos de estudo & 88 & $1,43 \%$ & 490 & $7,97 \%$ & 1476 & $24,01 \%$ & 4093 & $66,59 \%$ & 6147 & \\
\hline 8 a 12 anos de estudo & 77 & $1,11 \%$ & 203 & $2,92 \%$ & 1795 & $23,82 \%$ & 4877 & $70,15 \%$ & 6952 & \\
\hline$>12$ anos de estudo & 62 & $2,31 \%$ & 403 & $14,99 \%$ & 439 & $16,33 \%$ & 1785 & $66,38 \%$ & 2689 & \\
\hline Ignorado & 355 & $1,39 \%$ & 2215 & $8,66 \%$ & $\$ 096$ & $31,66 \%$ & 14907 & $58,29 \%$ & 25573 & $<0,001$ \\
\hline
\end{tabular}

\section{Raça cor da pele}

\begin{tabular}{l|cc|cc|cc|cc|c|c}
\hline & $N$ & $\%$ & $\mathbf{N}$ & $\%$ & $\mathbf{N}$ & $\%$ & $\mathbf{N}$ & $\%$ & & \\
negra & 227 & $7,37 \%$ & 1403 & $45,52 \%$ & 141 & $1,15 \%$ & 10535 & $341,82 \%$ & 12306 & \\
branca & 184 & $3,92 \%$ & 921 & $19,64 \%$ & 93 & $0,98 \%$ & 8338 & $177,78 \%$ & 9536 & \\
outros & 2 & $0,03 \%$ & 24 & $0,36 \%$ & 3 & $1,49 \%$ & 172 & $2,58 \%$ & 201 & $<0,001$ \\
Ignorado & 201 & $4,19 \%$ & 1386 & $28,92 \%$ & 727 & $6,06 \%$ & 9674 & $201,84 \%$ & 11988 & \\
\hline TOTAL (\% linha) & $\mathbf{6 1 4}$ & $\mathbf{7 3} \%$ & $\mathbf{3 7 3 4}$ & $\mathbf{4 4 2} \%$ & $\mathbf{1 5 6 0 2}$ & $\mathbf{1 8 4 9} \%$ & $\mathbf{2 8 7 1 9}$ & $\mathbf{3 4 0 3} \%$ & $\mathbf{4 8 6 6 9}$ & \\
\hline
\end{tabular}

* Outras hepatites virais. Fonte: Autores, com base de dados do SINAN (2021).

Observou-se que a infecção pelo vírus no sexo masculino é predominante com 2277 (61\%) das notificações e o feminino apresentou 1457 (39\%), para o HAV. Corroborando com os dados, segundo o Boletim Epidemiológico, no período de 2009 a 2019, ressalvando que a dimensão no território nacional de casos de hepatite A, do sexo masculino, foi de 55,3\%, e no sexo feminino, de 44,7\%. (Brasil, 2020)

Além disso, o sexo masculino também foi maioria, quando comparado ao feminino, nas co-infecções e nas outras hepatites virais, com 345 (56,2\%) e 1469 (51,2\%) notificações, respectivamente. A taxa de notificação do sexo feminino na coinfecção foi de $269(43,8 \%)$ e das outras hepatites virais foi de $14027(48,8 \%)$.

No Rio de Janeiro, no período analisado, a principal provável fonte de contágio foi alimento/ água contaminado com o vírus da hepatite A, com 2295 (97,41\%) casos, seguido pela via domiciliar com 119 (52,65\%) notificações (tabela 2). Corroborando, Ferreira e Silveira (2004) a via mais comum de transmissão do vírus da hepatite A é pelo contato íntimo e prolongado com os indivíduos portadores da infecção, sendo o convívio familiar o maior fator de risco, porém, cerca de metade dos casos não é possível identificar a fonte de contágio. Para Silva, et al. (2007) a falta de água encanada, coleta de lixo e serviço de limpeza e canais de esgoto parcialmente obstruídos por resíduos sólidos provocando inundações, são facilitadores na transmissão da doença.

Porém, a via transfucional é a principal via da provável fonte de contágio para as co-infecção e as outras hepatites virais, com $58(1,85 \%)$ e $3015(96,05 \%)$ notificações, respectivamente. (Tabela 2) 
Tabela 2 - Distribuição das prováveis fontes de contágio das notificações dos casos de hepatite A, no Rio de Janeiro, 2010 à 2018. ( $p$ valor $<0,001)$.

\begin{tabular}{|c|c|c|c|c|c|c|c|c|c|c|}
\hline \multirow[t]{2}{*}{ Provável fonte de contágio } & \multicolumn{2}{|c|}{ Co-infecção } & \multicolumn{2}{|c|}{ Hepatite A } & \multicolumn{2}{|c|}{ Ignorado } & \multicolumn{2}{|c|}{ Outros* } & \multirow{2}{*}{ Total } & \multirow[t]{2}{*}{ P valor } \\
\hline & $N$ & $\%$ & $\mathbf{N}$ & $\%$ & $\mathbf{N}$ & $\%$ & $\mathbf{N}$ & $\%$ & & \\
\hline Acidente de trabalho & 3 & $3,00 \%$ & 0 & $0,00 \%$ & 0 & $0,00 \%$ & 97 & $97,00 \%$ & 100 & \\
\hline Alimento/ água contaminado & 17 & $0,72 \%$ & 2295 & $97,41 \%$ & 24 & $1,02 \%$ & 20 & $0,85 \%$ & 2356 & \\
\hline Domiciliar & 2 & $0,88 \%$ & 119 & $52,65 \%$ & 0 & $0,00 \%$ & 105 & $46,46 \%$ & 226 & $<0,001$ \\
\hline Hemodiálise & 7 & $3,55 \%$ & 0 & $0,00 \%$ & 0 & $0,00 \%$ & 190 & $96,45 \%$ & 197 & \\
\hline Ignorado & 436 & $1,17 \%$ & 1161 & $3,11 \%$ & 15348 & $41,09 \%$ & 20411 & $54,64 \%$ & 37356 & \\
\hline Outros & 9 & $2,29 \%$ & 15 & $3,82 \%$ & 0 & $0,00 \%$ & 369 & $93,89 \%$ & 393 & \\
\hline Pessoa/pessoa & 1 & $0,35 \%$ & 94 & $33,10 \%$ & 0 & $0,00 \%$ & 189 & $66,55 \%$ & 284 & \\
\hline Sexual & 34 & $1,49 \%$ & 21 & $0,92 \%$ & 66 & $2,90 \%$ & 2158 & $94,69 \%$ & 2279 & \\
\hline Transfusional & 58 & $1,85 \%$ & 6 & $0,19 \%$ & 60 & $1,91 \%$ & 3015 & $96,05 \%$ & 3139 & \\
\hline Tratamento cirúrgico & 18 & $2,47 \%$ & 0 & $0,00 \%$ & 0 & $0,00 \%$ & 712 & $97,53 \%$ & 730 & \\
\hline Tratamento dentário & 14 & $2,12 \%$ & 18 & $2,73 \%$ & 0 & $0,00 \%$ & 627 & $95,14 \%$ & 659 & \\
\hline Uso de drogas & 13 & $1,65 \%$ & 4 & $0,51 \%$ & 38 & $4,82 \%$ & 733 & $93,02 \%$ & 788 & \\
\hline Vertical & 2 & $2,11 \%$ & 0 & $0,00 \%$ & 0 & $0,00 \%$ & 93 & $97,89 \%$ & 95 & \\
\hline TOTAL (\% linha) & 614 & $1 \%$ & 3734 & $8 \%$ & 15602 & $32 \%$ & 28719 & $59 \%$ & 48669 & \\
\hline
\end{tabular}

* Outras hepatites virais. Fonte: Autores, com base de dados do SINAN (2021).

\section{Considerações Finais}

A hepatite A, no ano de 2014, passou a fazer parte do Programa Nacional de Imunização (PNI), sendo administrada em dose única a partir dos 15 meses de vida da criança (Brito \& Souto, 2020). Porém, a doença ainda está presente no Brasil e sua abordagem é de extrema valia.

Entre os anos de 2010 e 2011 houve um aumento de 1,4\% nas taxas de notificações para o vírus da hepatite A, e em 2012 o aumento foi de 4,4\%. No ano de 2015, quando comparado ao ano anterior, houve queda na taxa de 6,2\% no número de notificações. Sendo que a menor prevalência da doença foi no ano de 2016 , com $0,7 \%$ dos casos, seguida de aumento de $3,7 \%$ em 2017 e de 8,7\% em 2018. (Gráfico 1)

A escolaridade mostrou-se relevante para os indivíduos reagente ao HAV, pois pessoas com menos de 8 anos de estudos são as mais acometidas pela doença. Nas co-infecções e outras hepatites virais, indivíduos que possuem mais de 12 anos de estudo apresentam o menor número de casos da doença. Isso, possivelmente, indica que indivíduos com uma escolaridade menor apresenta menos instrução sobre a doença e a sua forma de prevenção.

Foi observado que a principal fonte de contágio do HAV foi alimento/água contaminados, com 61,5\% dos casos. Contudo, novos estudos devem ser realizados para melhor compreensão da qualidade da água ofertada para a população e da efetividade no seu acesso, uma vez que a doença é transmitida, principalmente, por alimento/água portadores do vírus.

A faixa etária $\leq 24$ anos foram de maior notificação para o HAV, assim, são os jovens os mais acometidos pela hepatite A. Tal fato pode ser relacionado as crianças, de idade escolar, por serem mais suscetíveis a contrair a doença e a transmiti-la, uma vez que elas tendem a ser assintomáticas e frequentam lugares fechados, como creche e escolas, que facilitam a propagação da doença.

Ademais, segundo os dados analisados, o vírus da hepatite A acomete mais indivíduos da raça negra. O HAV por estar, principalmente, associado a falta de acesso ao saneamento básico, água potável e informação da doença, pode ter como hipóteses que a população negra, em sua maioria, viva em condições de precariedade. 
A taxa de notificações ignoradas para os reagentes ao HAV na variável escolaridade é alta, apresentando 8,66\% das notificações. O mesmo ocorre com a variável raça/cor, o qual apresenta a taxa de ignorado de 12,31\%. Desta forma, o presente estudo encontrou as notificações ignorados como a principal limitação, pois a ausência de informação dificulta a compreensão do perfil dos indivíduos notificados e interfere no planejamento de estratégias de saúde pública e na gestão. Assim sendo, a notificação de forma correta e completa é de extrema importância para a melhora dos serviços prestados.

Sugere-se a realização de novos estudos para melhor compreender o perfil epidemiológico da hepatite A nas demais regiões do Brasil, estratégias sanitárias para minimizar os danos à saúde da população e como melhorar a conscientização dos profissionais de saúde da importância da notificação, de forma correta, da Ficha de Investigação das Hepatites Virais. Ademais, o presente estudo tem o intuito de estimular a conscientização da importância do saneamento básico na prevenção da hepatite A.

\section{Referências}

Araújo, M. M. P. \& Silva, C. G. (2015). A importância do sistema de informação de agravos de notificação - SINAN para a vigilância epidemiológica do Piauí. Revista Interdisciplinar Ciência e Saúde. 201, 25-29.

Bandeira D. M. (2017). Etiologias de casos de hepatites agudas e perfil epidemiológico dos casos de hepatite A atendidos no Ambulatório de Hepatites Virais da FIOCRUZ 1997 a 2015. Dissertação de mestrado. Instituto Oswaldo Cruz.

Barrocas, P. R. G., Moraes, F. F. M. \& Sousa, A. C. A. (2019). Saneamento é saúde? O saneamento no campo da saúde coletiva. História, Ciências, SaúdeManguinhos. 26(1), 33-51.

Bordalo, A. A. (2006). Estudo transversal e/ou longitudinal. Revista Paraense de Medicina. 20(4).

Brasil. Ministério da Saúde. Secretaria de Vigilância em Saúde. Departamento de DST, Aids e Hepatites Virais. (2009). ABCDE do diagnóstico para as hepatites virais; 24p. Brasília (DF).

Brasil. Ministério do Desenvolvimento Regional. Secretaria Nacional de Saneamento. (2019). 24 Diagnósticodos Serviços de Água e Esgotos. Sistema Nacional de Informações sobre Saneamento - SNIS; 183p. Brasília (DF).

Brasil. Ministério da Saúde. Secretaria de Vigilância em Saúde. (2020). Boletim Epidemiológico das Hepatites Virais; 79p. Brasília (DF).

Brito, W. I. \& Souto, F. J. D. (2020) Vacinação universal contra hepatite A no Brasil: análise da cobertura vacinal e da incidência cinco anos após a implantação do programa. Rev Bras Epdemiol, 23: E200073. 10.1590/1980-549720200073

Chaves, G. C., Castro, C. G. S. O. \& Oliveira, M. A. (2017). Compras públicas de medicamentos para hepatite C no Brasil no período de 2005 a 2015. Cien Saude Colet, 22(8): 2527-2538.

Ferreira, V. M., Gonçalves, E. \& Gonzaga, L. M. O. (2017). Hepatites virais: epidemiologia dos casos notificados no estado de Minas Gerais entre 2005 e 2014. Revista Unimontes Científica, 19(1): 70-78.

Ferreira, C. T. \& Silveira, T. R. (2004). Hepatites virais: aspectos da epidemiologia e da prevenção. Rev. Bras. Epidemiol, 7(4): $473-87$.

Guimarães, L. C. C., Brunini, S., Guimarães, R. A., Júnior, H. G., Minamisava, R., Cunha, V. E., Santos, J. R. S., Lacerda, E. P. S., Souza, C. M., Oliveira, V. L. B., Albernaz, G. C., Menezes, T. G. \& Rezza, G. (2019). Epidemiology of hepatitis B virus infection in people living in poverty in the central-west region of Brazil. BMC Public Health, 19(1):443. 10.1186/ s12889-019-6828-8.

Ministério da Saúde. (2017). Hepatites virais. Guia de Vigilância em Saúde: volume único. Ministério da Saúde.

Ministério da Saúde - MS. (2018). Prevenção e Controle das IST, do HIV/Aids e das Hepatites Virais. Manual Técnico para o Diagnóstico das Hepatites Virais. Ministério da Saúde.

Nunes, F. S., Facco, L., Fecury, A. A., Araújo, M. H. M., Oliveira, E., Dendasck, C. V., Souza, K. O. \& Dias, C. A. G. M. Número de casos confirmados de hepatites virais no Brasil entre os anos de 2010 a 2015. Revista Científica Multidisciplinar Núcleo do Conhecimento. 25(11), 71-80..

Jesus, V. (2020). Racializando o olhar (sociológico) sobre a saúde ambiental em saneamento da população negra: um continuum colonial chamado racismo ambiental. Saúde Soc: 29(2), e180519. 10.1590/S0104-12902020180519

Junior, M. J. (2015). Como escrever trabalhos de conclusão de curso: instruções para planejar e montar, desenvolver, concluir, redigir e apresentar trabalhos monográficos e artigos, (9a ed.) Ed Vozes, 247p.

Pereira, F. E. L. \& Gonçalves, C. S. (2003). Hepatite A. Revista da Sociedade Brasileira de Medicina Tropical, 36:387-400.

Petruccelli, J. L. \& Saboia, A. L. (2013). Caracteristícas étnico-raciais da população: classificações e identidades: Instituto Brasileiro de Geografia e Estatística. 
Research, Society and Development, v. 10, n. 4, e28810414212, 2021

(CC BY 4.0) | ISSN 2525-3409 | DOI: http://dx.doi.org/10.33448/rsd-v10i4.14212

Silva, P. C., Vitral, C. L., Barcellos, C., Kawa, H., Gracie, R. \& Rosa, M. L. G. (2007). Hepatite A no Município do Rio de Janeiro, Brasil: padrão epidemiológico e associação das variáveis sócio-ambientais. Vinculando dados do SINAN aos do Censo Demográfico. Cad. Saúde Pública, 23(7), 15531564 